\title{
XXXIII.
}

\section{Aktive Immunisierung oder histogene Chemotherapie?}

\author{
Erwiderung an die Professoren F. Blumenthal und C. Lewin. ${ }^{1}$ ) \\ Voll
}

Dr. G. Fichera.

Die Forscher auf dem Gebiete der Geschwulstlehre kennen die um das Jahr 1902 von der Berliner Schule ausgeführten therapeutischen Versuche: v. Leyden, F. Blumenthal, C. Lewin, von der Annahme des Meisters über den parasitären Ursprung der Geschwülste ausgehend, leiteten mit virulentem Material (frisches neoplastisches Gewebe) die Vakzinotherapie (Auslösung einer aktiven Immunität) der Geschwülste ein.

Die ersten Vorversuche sowie die Methode und der Zweck der Untersuchungen sind deutlich niedergelegt in den Arbeiten ${ }^{2}$ ) dieser Forscher sowie in dem in Nr. 24 der "Deutschen medizinischen Wochenschrift" 1903 erschienenen zusammenfassenden Bericht ${ }^{3}$ ) über die Tätigkeit der der ersten medizinischen Klinik der Charité angegliederten Abteilung für Krebsforschung.

1) F. Blumenthal und C. Lewin, Weitere Versuche über Behandlung von Sarkomratten mit den Extraktstoffen des eigenen Tumors. Therapie der Gegenwart. 1914. H. $\ddot{3}$.

2) von Leyden und Blumenthal, Vorläufige Mitteilungen über einige Ergebnisse der Krebsforschung auf der I. med. Klinik. Deutsche med. Wochenschr. 1902. Nr. 36.

3) Ernst von Leyden ist bekanntlich auf Grund jahrelanger Forschungen Anhänger der parasitären Richtung der Entstehung des Carcinoms. Fr ist für die Ansicht in 2 Vorträgen 1901 und 1902 eingetreten. In diesen Vorträgen hat er die Gründe mitgeteilt, welche ihn bewogen, gewisse, bestimmter charakterisierbare Formen der Zelleinschlüsse in den Tumoren für die Krebsparasiten anzusprechen.

Ein grosser Teil des Laboratoriums soll in den Dienst serumtherapeutischer Untersuchungen gestellt werden, welche vor mehr als 2 Jahren von E. v. Leyden und F. Blumenthal begonnen und über die im vorigen Jahre in dieser Wochenschrift eine vorläufige Mitteilung erschienen ist.

Von v. Leyden und Blumenthal ist aber noch ein zweiter Weg zur Behandlung der Krebskranken eingeschlagen worden. Nach Art der Tuberkulinbehandlung haben sie mit dem zerriebenen Tamor selbst den kranken Menschen zu immunisieren versucht, wie sie das in ihrer vorläufigen Mitteilung genauer erwäbnen. 
Diese Anschauungen über parasitären Ursprung und Vakzinotherapie der Geschwülste kommen neuerdings zum Ausdruck in den nach einer längeren Unterbrechung von Blumenthal und Lewin wieder aufgenommenen Arbeiten. So wiederholt Lewin in seinem Werke über Geschwülste (1909): „Was wir aber durch das Experiment für erwiesen halten, ist, dass es unbekannte parasitäre Gebilde gibt, welche zur Entstehung maligner Geschwülste führen können", und andererseits kommt auch Blumenthal (1910) nochmals auf die "aktive Immunisierung" der mit experimentellen Sarkomen belasteten Ratten mittels "Autovakzine" zurück.

Das sind in ihren Grundzügen die Theorie und die Forschungen der v. Leydenschen Schule; diesen gegenüber kann behauptet werden: dass die bisher nicht bewiesene parasitäre Natur der Geschwïlste im eigentlichen Sinne des Wortes von den meisten Forschern in Abrede gestellt wird; dass die erworbene Resistenz der Spezifität entbehrt, da sie in mehr oder weniger hohem Grade durch die verschiedensten, neoplastischen oder normalen, embryonalen oder ausgewachsenen Gewebe verliehen wird; dass von einer wirklichen Immunisierung weder beim tumortragenden Menschen noch beim Tiere die Rede sein kann (Bashford, Haaland, Fichera); dass die Behandlung mit frischem Tumorgewebe eine Infektionsquelle für den Kranken darstellt (Delbet 1910, v. Graff und Ranzi 1912), ein Uebelstand, der bereits vorausgesehen und umgangen wurde (Fichera 1909).

Noch ehe Blumenthal und Lewin im Dezember $1910^{1}$ ) die neue Phase ihrer Untersuchungen mit einer Note einleiteten, in welcher der erstere, immer unter Bezugnahme auf aktive Immunisierung und Autovakzination, über die Heilung eines Rattensarkoms nach Einspritzungen neoplastischer Autolysate berichtete, hatte ich in mehreren Artikeln die Resultate meiner Untersuchungen niedergelegt, die seit 1907-1908 begonnen und unter Einhaltung bestimmter Prinzipien systematisch durchgeführt waren ${ }^{2}$ ).

Die vergleichend verfolgte Uebertragung embryonaler und neoplastischer Gewebe, das Studium der Verhältnisse, welche deren Verlauf fördernd oder hemmend beeinflussen, die Bestimmung der hauptsächlichsten, die Biologie der Geschwülste betreffenden, pathologisch-anatomischen, physio-pathologischen und statistischen Daten, haben mich seit langem zum Aufbau der Theorie des onkogenen Gleichgewichtsmangels und der histogenen Therapie, in bezug zur Genesis und Behandlung bösartiger Geschwiilste, geführt.

Meine Versuche brachten mich zur Anstellung und Begriindung eines

1) Blumenthal, F., Ueber Heilungsversuche bei einem Rattensarkom. Med. Klinik. 1910. Nr. 50.

2) Apolant, Die experimentelle Erforschung der Geschwülste. Jena 1913, G. Fischer. Vor allem hat sich aber Fichera eingehend mit der Wirkung von Autolysaten beschäftigt. 
Vergleiches zwischen der durch Embryonengewebe und der durch Geschwulstgewebe verliehenen Resistenz gegen weitere homologe oder gekreuzte Gewebseinführungen, was unter anderen auch von Krongold in einer unlängst im Institut Pasteur verfassten Thesis bestätigt wurde. Ganz besonderes Augenmerk schenkte ich ferner dem Zusammenhang zwischen Resorption der zuerst eingeimpften Geschwulst und erworbener Resistenz gegen eine spätere Uebertragung ${ }^{1}$ ).

In der Tat führten mich schon anfangs eine Reihe von Tatsachen, die ich Fall für Fall beleuchtete, zur Ueberzeugung, dass hier nicht von einer Immunität im gewöhnlichen Sinne des Wortes die Rede sein könne, dass es sich weder um spezifische Antikörperbildung noch um dauernde Unempfindlichkeit handele; ich vertrat die Meinung, dass die Rückbildung. der embryonalen wie der neoplastischen Uebertragung an eine Autolyse in vivo gebunden sei, mit direkter Befreiung und indirekter rascher, aber vorübergehender Bildung von dem lmpfprodukt schädlichen Substanzen²).

1) Krongold, Recherches expérimentales sur les greffes embryonnaires. Thèse. Paris 1914. L'immunisation cellulaire conférée à des animaux néoplasiques suggéra a quelques auteurs l'idée d'en emprunter la méthode pour faire une étude complète sur la greffe du tissu embryonnaire, l'immunité et la greffe n'étant que les termesconnexes du mème problèmeainsiquel'a dit Ficheraqui essaya le premier, par le procédé des greffes en série, de rendre réfractaires des rats à la greffe de tissus embryonnaires.

2) G. Fichera, Etiologia del cancro. 1909. Beim Studium der Wirkung histogener Autolysate, in der Absicht den direkten Mechanismus durch onkolytische Isound Heterofermente näher zu kennzeichnen, den ich zusammen mit dem indirekten Mechanismus, d. h. durch Reizung seitens Organen, welche die GeschwulstentwickIung hemmend beeinflussen, studiert hatte, drückte ich mich folgendermassen aus:

„Ich habe zuerst hervorgehoben, dass die langsame spontane Involution der eingeführten Embryonengewebe und der wenig virulenten Neoplasmazellen einerseits, sowie andererseits der rasche, nach Einspritzung von Autolysaten frühzeitig erfolgende Rückgang der embryonalen Gewebe und der höchst virulenten Geschwulstarten, gleiche morphologische Merkmale besitzen, die eine Erklärung ihrer Wirkungsweise ermöglichen.

Es handelt sich in jedem Falle um spezifische, ausgedehnte, cytolytische Erscheinungen, die von einer konnektiven und vaskulären Substitution gefolgt und begleitet sind. Diese Erscheinungen stellen sich bei weniger aktiv wuchernden Geweben zwar spät, aber immerhin spontan ein; sie zeigen bei energisch sich entwickelnden Geweben sich hingegen spärlich und unzulänglich, können aber durch Einführung autolytischer Produkte angeregt werden.

Gedenkt man nun der Fermente, welche die in Zerstörung begriffenen Stellen im Impftumor zurücklassen, so findet man leicht eine Erklärung für đie spontane und auch für die durch Einführung von an freien Fermenten reichen Autolysaten erzeugte Rückbildung.

Es besitzen dieselben eine ausgesprochene Wirkung auf die übertragenen 
Derartige Befunde und Folgerungen dienten mir zur Richtschnur für therapeutische Versuche ${ }^{1}$ ), bei denen ich die Autolyse in vitro sich ab-

Gewebe, was aus dem histologischen Studium nicht nur der eingeführten embryonalen und neoplastischen Gewebe, sondern überhaupt auch aus dem aller übrigen Zellen des organischen Haushaltes ersichtlich ist.

$\mathrm{Z}$ wischen autolytischen Produlten und eingeführten, in Wucherung begriffenen Zellenelementen bildet sich sozusagen ein elektiver Bund, demzufolge sie nur bis zur vollständigen Zytolyse angegriffen und geschädigt werden. Intensität und zeitlicher Verlauf der Zytolyse sind einesteils von der Bildung der Zellfermente, andernteils von der Empfindlichkeit der histologischen Fermente ihr gegenüber abhängig.

Ob es sich hier um ein direktes oder indirektes Verhältnis bandelt, ist schwer zu entscheiden.

Die Wirkung der antolytischen Produlite kommt nach Finführung in den Gesamtorganismus rasch zum Ausdruck, wobei keine Läsion an der Einführungsstelle zu beobachten ist, wohl aber ein dentlicher Ënfluss auf die experimentelle Geschwulst; bei Einspritzung in das Geschwulstgewebe selbst tritt sie aber noch rascher, sogar plötzlich in Erscheinung, indem längs den mit der Nadel in Berührung kommenden Gewebsflächen eine ausgedehnte enzymatische Nekrose zu beobachten ist. Auf Grund solcher Bemerkungen ist die Annahme einer direkten Alition logischer als die einer durch die eingespritzten Substanzen ausgelösten Antikörperbildung.

Gegen die letztere Hypothese sprechen die konstant negativ ausfallenden Versuche, durch Immunisierung mit Geschwulstextrakten ein Immunserum za gewinnen, sowie auch die Prüfungen des Serums von Tieren, die mit Geschwulstgeweben behandelt oder geheilt worden waren.

Wie ich bereits erwähnte, ist mir in keinem Falle von Geschwulstinvolution, es sei dieselbe auf irgendeine beliebige Weise erfolgt, der Nachweis von Antikörpern gelungen. Es sei hier noch angefügt, dass ich im gleichen Sinne die Versuche deute, die für das Fehlen einer den Autolysateinspritzungen innewohnenden Schntzwirkung sprechen, sowie jene, welche die kurze Dauer der Widerstandsfähigkeit beweisen und aus denen ersichtlich ist, dass die in den Organismus eingeführten oder dort gebildeten autolytischen Produkte wieder ausgeschieden werden, falls sie nicht auf ähnliche, geeignete Elemente stossen, mit denen sie in den Kampf eingehen können.

Solche Versuche lassen die Bildung spezifischer Antikörper ausgeschlossen oder zum mindesten nicht bewiesen erscheinen; sie bestätigen zugleich die Wirkang besonderer Zellfermente, welche aus der Geschwulst des Gasttieres spontan in Freiheit gesetzt werden oder mit den Einspritzungen dorthin gelangen."

1) G. Fichera, Sulla biologia dei tessuti embrionali e dei neoplastici. Gazz. degli osp. e clin. 1910. Nr. 109. Beim Bericht über die seit mehr als Jahresfrist beim Menschen erhaltenen Resultate, welche unter Verwertung der von vornherein mit autolytischen Produkten von mir am Tier erzielten Ergebnisse angestrebt wurden, heisst es:

„Für den Versuch am Menschen musste aber aufjeden Fallein 
spielen liess, so dass mit der auf einer vorausgehenden Uebertragung folgenden Resistenz ein Teil des beobachteten Mechanismus ermöglicht und beschleunigt wurde und dem Organismus sofort, unter Ausschluss jeder Impfgefahr, eine erforderliche Dosis der onkolytischen Prinzipien zugeführt wird und überdies die Reizung zur Reaktion mit antiblastischen Substanzen, welch letztere gänzlich fehlen oder infolge der funktionellen Erschlaffung der durch individuellen Zyklus in Rückbildung oder Atrophie begriffenen Organe verarmt sein können.

Die zahlreichen Versuchsreihen, welche von mir und einigen Mitarbeitern im Hinblick auf die obigen Anschauungen am Tiere sowie am Menschen durchgeführt wurden, sind leicht aus der Literatur zu ersehen ${ }^{1}$ ).

Ich glaubte demnach, dass die Richtung der v. Leyden-, Blumenthal- und Lew inschen Untersuchungen, schon nach den klaren, bestimmten Aussagen der letzten beiden Forscher zu schliessen, deutlich von jener der meinigen sich unterscheide: In dieser Ueberzengung stützten mich, ausser dem Tatsachenmaterial der Vergangenheit, namentlich die auf dem Dritten internationalen Kongress für Krebsforschung (Brüssel, August 1913) stattgefundene Diskussion, welcher Blumenthal ${ }^{2}$ ) beiwohnte, der übrigens auch auf dem zweiten Kongress (Paris, Oktober 1910) anwesend war, auf dem ich die bereits an anderer Stelle mitgeteilten und veröffentlichten Versuche wiederholte.

Abgesehen von einigen früheren zur Richtigstellung der wahren Sachlage von mir ausgegangenen Polemiken wollte es mir in der Tat scheinen, als sei die Wahrheit anerkannt worden: denn sowohl Blumenthal als Lewin erkannten 1912 bezüglich der Behandlung mit Autolysaten mir das Prioritätsrecht zu und sicherten somit, was die Methode anbelangt, den mir gebührenden Anteil; und noch bei derselben Gelegenheit und auch

Frsatzmittel für den embryonalen oder neoplastischen Gewebsbrei gefunden werden, da es nicht angängig schien, hier an diebei der subkutanen Einführung von Geschwulst-oder Embryonenfragmenten bei Tieren übliche Technik der Aushöhlung zu denken.

Die Gewebsautolysateder Ratten selbst schienen das Problem zu lösen, dabei deren Verwendung die hauptsächlichsten technischen Schwierigkeiten umgangen und auch einzeitlicher Vorteil gewonnen werdenkonnte, indem der Befreiungsprozess der aktiven Substanzen sich bereits in vitro vollzogen hat."

1) G. Fichera, Ausbau der Theorie des onliogenen Gleichgewichtsmangels und der histogenen Chemotherapie zur Entstehung und Behandlung der bösartigen Geschwülste. Zeitschr. f. Krebsforschung. 1914. Bd. 14. Tumori. 1913. Anno III. Fasc. 1.

2) Fichera, Contributo alla III Conferenza internazionale per il cancro. Tumori. 1913. Anno III. Fasc. 2. 
später, 1913, bestanden beide Forscher lebhaft auf einer Unterscheidung ihrer eigenen Anschauungen von den meinigen ${ }^{1}$ ), was mir für die persönliche Richtung meiner Versuche bürgte.

All dieses steht jedoch in einem eigentümlichen Kontrast mit einer eben erschienenen Note, in welcher die beiden Forscher zusammen, beim Bericht über die Heilung des Rattensarkoms mit den durch Fällung aus dem Autolysat gewonnenen aktiven Substanzen, das vergessen, was sie früher anfrecht gehalten und gleichzeitig mit wenigen Sätzen - vielleicht in der Unmöglichkeit, die eigenen Ideen weiter zu vertreten - die bisher befürworteten tiefgreifenden Unterschiede zu verwischen suchen, so dass an eine Verwechslung der beiden Systeme zu denken wäre.

Ich will zuerst nur die Tatsachen bringen und hernach näher darauf eingehen.

\section{2.}

C. Lewin, Immunisierungs- und Heilversuche mit AutoIysaten bei Rattentumoren. Zeitschr. f. Krebsforschung. 1912. Bd. 11. Heft 3.

„Fichera hat ebenfalls Heilversuche mit autolysierten Tumormassen angestellt und ebenso wie bei Verwendung autolysierter embryonaler Zellen nicht nur bei Rattensarkomen, sondern auch in einer grossen Reihe von menschlichen Tumoren sehr beachtenswerte Erfolge erzielt. Er berichtet darüber ausführlich in seinem Werke über Tumoren und beschreibt seine Versuche folgendermassen."

F. Blumenthal, Ueber die Rückbildung bösartiger Geschwülste durch die Behandlung mit dem eigenen Tumorextrakt (Autovakzine). Zeitschr. f. Krebsforschung. 1912. Bd. 11. Heft 3.

"Nach dieser Veröffentlichung Jensens sind dann unabhängig von ihm angestellte Versuche an Ratten (Sarkom) von Fichera ${ }^{2}$ ) mitgeteilt worden."

\section{4.}

Weitere Versuche uber Behandlung von Sarkomratten mit den Extraktstoffen des eigenen Tumors, von Prof. Dr. Ferdinand Blumenthal und Prof. Dr. Carl Lewin. Die Therapie der Gegenwart. März 1914. Heft 3.

"Es war daher von grösster Bedeutung, die Methode so zu

1) Lewin, C., Die Behandlung von Krebskranken mit Vakzination. Therapie der Gegenwart. 1913. H. 6. - Blumenthal, F., Der gegenwärtige Stand der Behandlung der bösartigen Geschwülste. Berliner klin. Wochonschr. 1913. Nr. 50.

2) Fichera, G., Etiologia del cancro. Arch. e atti della soc. Ital. di chirurg. XXII adem. Roma 1909. Arch. intern. de chir. Gand 1910. 
verbessern, dass einerseits eine Uebertragung von lebenden Krebszellen ausgeschlossen war, andererseits die Wirksamkeit der Extrakte nicht abgeschwächt wurde. Beides erreichte Blumenthal, indem er die Tumorextrakte ein bis drei Tage bei $39^{\circ}$ im Brutschrank stehen liess. Durch Versuche an Ratten wies er nach, dass so behandel te Extrakte keine neuen Tumoren erzeugen, und dass sie gleichzeitig ausserordentlich wirksam waren.

Diese Resultate konnte Carl Lewin im Berliner Krebsinstitut im Jahre 1911 bei verschiedenen Sarkomstämmen bei Ratten bestätigen"1).

Die Leser verfügen nun über die Elemente, um beurteilen zu können, ob eine Aneignung seitens der beiden Forscher vorliegt; es waren denselben meine vorausgegangenen Studien bekannt, sie hatten diese referiert und sogar eine der Quellen angegeben, wie aus dem Zitat des einen im Jahre 1909 hervorgeht.

Das Vorgehen Lewins zugunsten Blumenthals könte nun zwar Staunen erwecken, wenn man sich nicht uiberzeugen würde, dass seinerseits Blumenthal Lewin mit einem ähnlichen Versehen erwidert.

Um eine Verbindung zwischen den zwei Autoren zu ermöglichen, musste notgedrungen ein Teil der früheren Produktion Lewins übergangen werden, dessen Existenz dem Versuch, aus der Behandlung mit histogenen Autolysaten eine einfache Sache der Technik zu machen und aktive Immunisierung mit Histotherapie zu rerwechseln, im Wege gestanden liätte.

Blumenthal und Lewin sind nunmehr darin einig geworden, die unter den Namen ,aktive Immunisierung, Tumorautolysatbehandlung, Vakzine- oder Vakzinationstherapie" begriffenen Methoden als gleichbedeutend zu betrachten und in einer einzigen Bedeutung zu verschmelzen. Gegen eine solche Verschmelzung, die scheinbar nur die Terminologie betrifft, sprechen aber absolut hier verschwiegene Untersuchungen und Behauptungen Lewins: In der Tat sind die Immunisierungsversuche mit bereits autolysierten Geweben (die nicht die Phase der Resorption in viro abwarten) ebenso wie zuerst in den Händen von Fichera, Daels und Deleuze, so auch in denen von Lewin misslungen, so dass er diesbezüglich zu folgenden Schlüssen kam: „Eine Immunisierung von Ratten durch Injektionen von Autolysaten gegen spätere Impfungen ist mir bisher nicht gelungen. Es handelt sich bei diesen Versuchen Rovsings ebensowenig wie in den schon erwähnten Ver-

1) C. Lewin (1912): „Demnach hat auch das Autolysat von 4 bis 6 Wochen nach der Mothode Ficheras noch oine Heilwirkung ausgeäbt, die im ganzen ebenso stark ist wie die nach Blumenthals Angaben angewandte Versuchsanordnung der ersten Serie." 
suchen ron Blumenthal und v. Leyden um aktive Immunisierung, sondern wahrscheinlich um Wirkungen der. Fermente des autolysierten Tumors, so wie das auch Fichera betont" (1912).

Und als ob diese Versehen und diese Widersprïche an und für sich nicht ausreichten, gebe ich hier noch ein anderes schwereres Beispiel: Zur Stütze des Prinzips der Autovakzination, parallel mit der Immunisierung, hatte Blumenthal die Notwendigkeit einer völligen Uebereinstimmung in Art, Sitz, histologischem Typus zwischen dem in Behandlung stehenden und dem sogenannten immunisierenden Tumor betont; eben diese Anschauung unterschreiben nun die beiden Forscher. Es ist aber noch nicht lange her, dass Lewin beziglich der durch Impfung erworbenen Resistenz eine solche Notwendigkeit völlig in Abrede stellte: „Ich kann demnach nach meinen Frfahrungen bei Ratien nur schliessen, dass zwischen Carcinom und Sarkom in bezug auf Immunitat Wechselwirkungen bestehen, deren Art uns allerdings noch unbekannt ist."

Lew in wusste also, dass bei der durch Gewebseinfiuhrung verliehenen Widerstandsfähigkeit keine strenge Spezifität zu Recht bestehe, die den Begriff der Vakzination aufkommen liesse; eine Behauptung; die mich veranlasste, gegen Blumenthal auf dem Kongress zu Brüssel Einwand zu erheben, die in der Folge ron Bertrand, Lunckenbein, Shirlaw, Little, Barrat und Gelarie beanstandet wurde, und in jüngster Zeit endlich auch durch die Kontrollversuche mittels der Abderhaldenschen Nethode ${ }^{1}$ ).

Er konnte sich äbrigens nicht unbewusst sein, dass mit seiner obengenannten Zustimmung und in Einklang mit Erhardts²) späteren An-

1) Simon, Die Behandlung der Geschwülste. Berlin 1914, Springer. „A uch die Art, wie diese Immunitäterzielt werden kann, beweist deatlich, dass es sich um keinen streng spezifischen Vorgang handelt; die Immunität wird nämlich nicht nur bewirkt durch Vorbehandlung mit schwach virulentem Tumornaterial im Sinne einer echten aktiven Immunisierung, sondern es gelingt auch durch Einverleibung von Embryonenbrei, Leberzellen, Blutkörperchen und anderem beliebigem Zellmaterial der eigenen wie einer anderen Art, denselben Effektzu erzielen."

2) Erhardt, Münchenel med. Wochenschr. 1913. Nr. 27. „Die Beeinflussung der Tumoren durch Injektion von Tumorextrakten ist also wohl grösstenteils a uf die Wirkung der darin enthaltenen Fermente zur ückzuführen, eine Konstatierung, die deshalb von Wichtigkeit ist, weil man bei Anwendung ähnlicher Verfabren bei Menschen doch wohl Bedenken haben muss, die ganze Summe der in den Extrakten oder gar in den Avtolysaten rorhandenen Körper einem Organismus einzuverleiben." 
schauungen bereits durch mich die Erscheinungen meiner Theorie erklärt wurden, und zwar gerade mit einer Auslegung, die einen der Gesichtspunkte des heutigen neuen Begriffes der beiden Forscher darstellt.

In der Tat schreiben Blumenthal und Lewin an der Stelle, an der sie die Versuche Erhardts ${ }^{1}$ ) wiederholen, der mit dem alkoholischen Präzipitat der Autolysate die Riickbildung der Geschwiilste erzielte: „Das, was ausfält, sind im wesentlichen Eiweisskörper, welche entweder selbst die wirksame Substanz darstellen oder mit einschliessen."

Nach einer solchen Behauptung kann das eingehaltene Verfahren unmöglieh mit aktiver Immmisierung, mit Vakzinotherapie, im eigentlichen Sinne des Wortes in Einklang gebracht werden, um so weniger von Forschern, welche von Untersuchungen auszugehen behaupten, die sie im Jahre 1912 an der Hand der Tuberkulosebehandlung mittels Tuberkulin ausführten.

Ebensowenig ist es angängig, unter dem Vorwande technischer Modifikationen und angeblicher Verbesserungen der Methode bis dahin aufrecht gehaltene, grundsätzliche Unterschiede in Konzept und Ansfiihrung zu verbergen, die man auf einmal unversehens beseitigen möchte.

Augenscheinlich zeigt die heutige Note ron einer radikalen Wechselstimmung, die nicht verschleiert werden kann und darl und der die Autoren bis vor wenigen Monaten widerstanden haben.

Die Bewegung zugunsten des onkogenen Gleichgewichtsmangels und die Beweisführungen zu der damit verbundenen histogenen Chemotherapie sind aber in letzter Zeit derart angewachsen, dass sich auch die beiden alten Gegner dieser Lösung des Problems der Behandlung bösartiger Geschwülste nähem, die sie zwar fir richtig und praktisch halten, nicht aber mit ihrem ursprünglichen Konzept identifizieren können. Ton den in dieser Richtung wirkenden Forschern seien nur die letzten erwähnt, wie Adami, Gilford, Shirlaw, Kenneth, Little, Moulin, Pfahler, Theilhaber, Erhardt, Barratt und Gelarie, Lunkenbein, Abderlalden, Oeller, Stephan.

1) Blumenthal und Lew in schreiben die genannte Modifikation der Technik Frhardt zu; letzterer verschwieg aber die früber erschienenen Arbeiten von Daels und Deleuze. Diese beiden Forscher, die die ersten Bestätigungen meiner Befunde erbrachten, erklärten schon ein Jahr vor dem Erscheinen der Erhardtschen Arbeit (Arch. de méd. expérim. 1912.): „A ce dernier point de vue, la méthode de choix, pour l'isolement des ferments protéolytiques, serait l'isolement du ferment par précipitation alcoolique après 1--2 jours d'antolyse du tissu et antolyse de la solution du ferment durant deux semaines.

... Cette action nette du ferment de tissuembryonnaire sur le tissu sarcomateux nous démontre l'action bétérolytique exercée par les ferments protéolytiques de tissus normaux sur le tissu néoplastique." 
Und hiermit glaube ich die nötigen, das Problem streifenden Daten geliefert zu haben; ich hielt es für gerechtfertigt, die Aufmerksamkeit darauf hinzulenken, nicht so sehr in Anbetracht der Ueberraschungen, die Blumenthal und Lewin denen, die ihnen vorausgegangen, reserviert hatten, als in der Absicht, nicht mowillkürliche Verwechslungen und Versehen zu klären, die das Studium der Geschwülste erschweren könnten.

\section{Versehen oder Plagiat?}

An Prof. K. Brandenburg.

Zur näheren Bestätigung und Erläuterung der Willkürlichkeit des Vorgehens, über das ich mich beklage, sehe ich mich genötigt, noch einen weiteren Beweis vorzubringen. Als Blumenthal im Dezember 1910, immer in der Absicht, die Ratten mit Sarkommaterial zu impfen, Herstellung und Verwendung der histogenen Autolysate entdeckt zu haben glaubte, schrieb ich eine Richtigstellung, die aber infolge der Vermittlung des Herausgebers der Zeitschrift „Medizinische Klinik“ in Deutschland nicht zur Veröffentlichung kam. Unter den Erklärungen, mit denen er sich ins Mittel legte, befand sich auch der folgende Satz: "Tatsache ist, dass Blumenthal Ihre Demonstration nicht gehört hat und ron Ihren Arbeiten zu der Zeit, als er seine Untersuchungen anstellte, keine Kenntnis gehabt hat. Man muss ihm also den Vorwurf machen, dass er die Literatur nicht genau studierthat, aber man kann ihm nicht vorwerfen, ein Plagiat begangen zu haben." (Brandenburg, 2. März 1911.) Nach dieser Aeusserung kann sich der Leser selbst ein Urteil bilden.

\section{Literaturverzeichnis.}

A. Aus dem Laboratorium der chirurgischen Universitätsklinik zu Rom (Prof. F. Durante), geleitet von G. Fichera (Juni 1913).

1. Fichera, G., Sugli innesti di tessuti embrionali e fetali. Il Policlinico, Sez. Prat. 1908; Arch. ed Atti Soc. Ital. di chirurgia, Congresso 1908.

2. Fichera, G., Sull'innesto di tessuti embrionali e fetali durante la gravidanza. Policlinico, Sez. Prat. 1909; R. Accad. Med. di Roma. 1909.

3. Fichera, G., a) Sul comportamento di sede negli innesti di tessuti embrionali e fetali; b) Sull'immunizzazione di tessuti embrionali e fetali; c) Sulla biologia dei tumori spontanei dei ratti. Policlinico, Sez. Prat. 1909; R. Accad. Med. di Roma. 1909.

4. Fichera, G., Parallelo fra l'innesto di tessuti embrionali e l'innesto di tessuti neoplastici. Policlinico, Sez. Prat. 1909; R. Accad. di Med. di Roma. 1909.

5. Fichera, G., Développement des greffes embryonnaires et foetales, immunisation qu'elles déterminent. Arch. de Méd. exp. et d'Anat. pathol. 1909.

6. Fichera, G., Etiologia del cancro. Policlinico, Sez. Prat. 1909; Atti Congr. Ital. di chir., 1909; Annali del R. Ist. di Chir. di Roma. 1910. 
7. Fichera, G., Primo contributo alla biologia dei tumori, Policl, Sez. Prat. 1910; R. Accad. Med. Roma. 1910; Bull. Assoo. Cult. scienze med. e natur. di Roma, in Arch. di Farmacol. sper. 1910.

8. Fichera, G., Secondo contributo alla biologia dei tumori. Bull. Assoc. Cult. scienze med. e natur. di Roma, in Arch. di Farm. sper. 1910.

9. Fichera, G., Terzo contributo alla biologia dei tumori. Bull. Assoc. Cult. scienze med. e natur. di Roma, in Arch. di Farm. sper. 1910.

10. Fichera, G., L'azione dei prodotti di autolisi fetali omogenei sui tumori maligni dell' uomo. Policlinico, Sez. Prat. 1910.

11. Fichera, G., Sulla biologia dei tessuti embrionali e dei neoplastici. L'azione dei prodotti di autolisi omogenea sui tumori maligni. Il. Interr. Konferenz über Krebsforschung. Pathologica, 1910.

12. Fichera, G., Sulla biologia dei tessuti embrionali e dei neoplastici. Gazz. degli Osped. 1910.

13. Fichera, G., Studi comparativi sulla biologia degli innesti embrionali e neoplastici. L'azione dei prodotti di autolisi omogenea sui tumori maligni. II. Konferenz über Krebsforschung. Pathologica, 1910.

14. Fichera, G., Tumori: Etiologia, Terapia. Torina, Unione Tip. Edit. 1911.

15. Fichera, G., Gli autolisati di tessuti nella terapia dei tumori. Policl. Sez. Prat. 1911. Pathologica, 1911.

16. Fichera, G., Ancora sugli autolizzati di tessuti nella terapia dei tumori. Policl., Sez. Prat. 1911.

17. Fichera, G., Chemioterapia istogena deitumori maligni. Kongress für Ḱrebsforschung, Dresden 1911.

18. Ficbera, G., Sui recenti contributi alla dottrina dei neoplasmi. Policl. Sez. Chir. 1911; 'Tumori, anno I. 1911.

19. Fi chera, G., Stato attuale della lotta contro il cancro. Tumori, annoll. 1913.

20. Fichera, G., Controllo e critica in oncologia. Tumori, anno II. 1913.

21. Fichera, G., Evoluzione della teoria del disquilibrio oncogeno e della chemoterapia istogena, per la genesi e la cura dei tumorimaligni. Tumori, annolli. 1913.

22. Fichera, G., Contributi alla III. Conferenza per il cancro. Tumori, anno III. 1913.

23. Fichera, G., Rassegna bibliografica. 'Tumori, fasc. 1-6. 1911-1912.

24. Ficbera, G., Rassegna bibliografica. Tumori, fasc. 1-6. 1912-1913.

25. Gussio, Sulla metamorfosi sarcomatosa dei carcinomi sperimentali. Gazz. int. di Med. e di Chir. 1911.

26. Gussio, Sul potere oncolitico nei ratti. Policl., Sez. Prat. 1911. Tumori, fasc. 1. 1911.

27. Gussio, Sviluppo neoplastico tipico e resistenza dell'ospite. Tumori, fasc. 2, 3. 1911.

28. Gussio, Trasmissione neoplastica atipica e difesa dell' ospite. Tumori, faso. 3, 4. 1911-1912.

29. Gussio, Trasmissione neoplastica e recettivitì d'età, di razza di specie. Tumori, fase. 2-4. 1912-13.

30. Gussio, Intorno all comportamento della massa sanguigna nei ratti con sarcoma da innesto. T'umori, fasc. 4. 1912. 
31. Brancati, Sul comportamento dei tumori innestati in varia sede. Tumori, fasc. 6. 1912.

32. Brancati, Sul comportamento della milza negli animali con tumore. Tumori, fasc. 6. 1912 .

33. Brancati, Il fegato nei ratti con tumore. Tumori, faso. 6. 1912.

34. Brancati, Sugli innesti di sarcoma in ratti splenectomizzati. Tumori, fasc. 5.1913.

35. Brancati, Sulmidollo osseo neiratticon tumore da innesto. Tumori, fasc.5. 1913.

36. Ricei, La milza nei cancerosi. 'Tumori, fase. 1. 1912.

37. Magnini, Sulla evoluzione degli innesti neoplastici nei ratti timectomizzati, Sullo stato del timo e la frequenza dei neoplasmi nelle varie età dell' uomo. Tumori, fasc. 3. 1912.

38. Magnini, Sull'azione patogena del saccharomyces neof. S. Tumori, annoIII. 1913.

39. Cara v ani, L'azione degli autolizzati fotali e neoplastici studiata in rapporto alla dose negli animali normali e portatori di tumori maligni. Tumori, fasc.6. 1913.

\section{B. Beiträge aus anderen Instituten Italiens.}

40. Cimoroni, Ricerchesperimentali su un epitelioma del topo. Tumori, fasc. 4. 1912.

41. Cimoroni, Sulle modificazioni della milza e del fegato nei topi portatori di epitelioma. Tumori, fasc. 6. 1912.

42. Almagià, Ricerche sperimentali sul rapporto fra la funzione delle ghiandole sessuali e lo sviluppo di tumori. Bull. R. Accad. med. di Roma. 1912.

43. Almagià, Considerazioni ed esperienze sui tumori degli animali. Tumori, fasc. 6. 1913.

44. Centanni e Bröking, Sulle blastine. Tumori, fasc. 6. 1912.

45. Centanni, Sulle blastine. Tumori, fasc. 3. 1913.

46. Centanni, La dieta aviride per lo sviluppo dei tumori sperimentali. Tumori, fasc. 4. 1913.

47. Uffreduzzi, Sull'azione dell'autolizzato fetale sui trapianti dei tessuti adulti. Pathologica. 62. 1912.

48. Uffreduzzi, Innesti di tumori e autolizzato fetale e neoptastico. Tumori, fas. 4. 1913.

49. Perez, Carcinoma della Iaringe, atrofia della glandola tiroide. Tumori, fasc. 3. 1912.

50. Cuzzi, Tentativo di antosieroterapia nei tumori maligni inoperabili. Tumori, fasc. 5. 1912.

51. Betti, Contributo alla terapia istogena dei tumorimaligni. Tumori, fasc. 4. 1912. 52. Bazzocchi, Sulla istoterapia dei tumori maligni dell'uomo. Tumori, fasc. 3. 1913.

\section{Nachtrag.}

Während ich auf die Verölfentichung der obigen Note in der Zeitschrift für Krebsforschung wartete, erschjen der Aufsatz von Prof. Ferdinand BIumenthal (Bd. 14, Heft 2) mit Bemerkungen zu meinem Sammelreferat: "Ausbau der Theorie des onkogenen Gleichgewichtsmangels" (Bd. 14, Heft 1).

Eine vollständige Erwiderung, mit entscheidenden Dokumenten, erscheint in der Zeitschrift "Tumori“. Anno IV. Fasc. 2. 1914. 